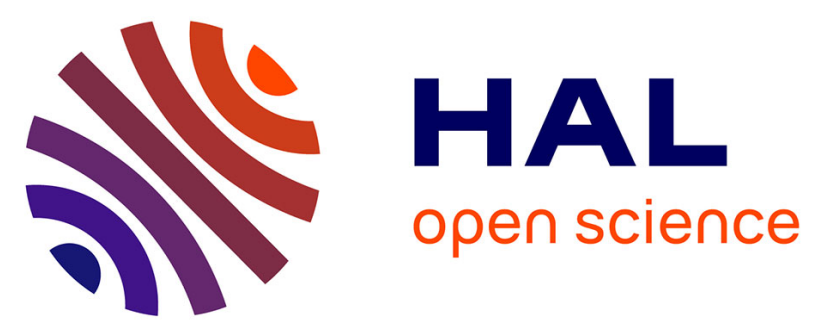

\title{
Equilibrium in an individual - societal SIR vaccination model in presence of discounting and finite vaccination capacity
}

\author{
Laetitia Laguzet, Gabriel Turinici, Ghozlane Yahiaoui
}

\section{To cite this version:}

Laetitia Laguzet, Gabriel Turinici, Ghozlane Yahiaoui. Equilibrium in an individual - societal SIR vaccination model in presence of discounting and finite vaccination capacity. Viorel Barbu, Cătălin Lefter, Ioan I. Vrabie. New Trends in Differential Equations, Control Theory and Optimization, World Scientific Publishing Co, pp.201 - 214, 2016, 978-9813142855. 10.1142/9789813142862_0015 . hal-01222450v2

\author{
HAL Id: hal-01222450 \\ https://hal.science/hal-01222450v2
}

Submitted on 4 Nov 2016

HAL is a multi-disciplinary open access archive for the deposit and dissemination of scientific research documents, whether they are published or not. The documents may come from teaching and research institutions in France or abroad, or from public or private research centers.
L'archive ouverte pluridisciplinaire HAL, est destinée au dépôt et à la diffusion de documents scientifiques de niveau recherche, publiés ou non, émanant des établissements d'enseignement et de recherche français ou étrangers, des laboratoires publics ou privés. 


\title{
Equilibrium in an individual - societal SIR vaccination model in presence of discounting and finite vaccination capacity
}

\author{
Laetitia Laguzet*, Gabriel Turinici*,** and Ghozlane Yahiaoui* \\ * CEREMADE, Université Paris Dauphine, Paris 75016, France \\ ** Institut Universitaire de France, \\ Tel.: +33-1-44054858 \\ E-mail: laguzet@ceremade.dauphine.fr, Gabriel.Turinici@dauphine.fr \\ ghozlane.yahiaoui@dauphine.eu
}

\begin{abstract}
This paper analyzes individual optimal vaccination strategies for a SusceptibleInfected-Recovered (SIR) model. The individuals vaccinate according to a criterion taking into account the risk of infection, the possible side effects of the vaccine and the overall epidemic course. We treat the situation when the vaccination capacity is limited and the individual discounts the future at some rate $\mathcal{D}>0$. Since the course of the epidemic is determined by the sum of individual decisions a natural question is whether an equilibrium between the individual decisions and the epidemic evolution exists. We show that the answer is positive.
\end{abstract}

Keywords: optimal vaccination, SIR model; mean field games.

\section{Introduction}

The vaccination, if available, is often invoked in order to limit the propagation of epidemics. A "benevolent planner" approach can be used to find the vaccination program that will strike the optimal balance between vaccination costs and benefits, expressed in terms of money, medical status, QALY/ DALY (see Refs. 1-3). Refs. 4-9 focused on describing the optimal vaccination policy as function of the vaccine cost, infection cost and epidemic dynamics.

However, failures in vaccination campaigns were observed due to worries related to the cost, risk, inefficiency or side-effects of the vaccine. The individual perception and choices will therefore depart from the "benevolent planner" approach and each individual will take his own best vaccination decision according to his subjective perceived risks and costs. As such other parameters should be taken into account and included in the model such as the individual point of view. Individual decisions, whether vaccination 
is accepted or not may influence the propagation of an epidemic. Several works analyzed this effect, see Refs. 10-20. We refer the reader to Ref. 21 for a recent literature review.

In this context, we use a SIR model and techniques from the "Mean Field Games" theory to show that an equilibrium exists and determines completely the individual optimal vaccination strategy. This work extends the analysis in Ref. 21 to the situation when the discount factor $\mathcal{D}$ is strictly positive but the vaccination capacity $u_{\max }$ is limited. We prove the existence (but not the uniqueness) of an equilibrium.

The work is close in spirit to Refs. 22,23 that show existence and uniqueness of the Mean Field Games equilibrium for a framework involving a finite number of possible states, but the hypothesis used there do not apply here (in particular the super-linearity of the cost with respect to the controls).

\section{Model and nomenclature}

We use a SIR model (see Refs. 8,24 for a general description and Ref. 21 for this particular model) described by the following equations:

$$
\begin{cases}S^{\prime}(t)=-\beta S(t) I(t)-u(t), & S(0)=S_{0} \\ I^{\prime}(t)=(\beta S(t)-\gamma) I(t), & I(0)=I_{0} \\ \varphi_{I}^{\prime}(t)=\left(1-\varphi_{I}(t)\right) \beta I(t), & \varphi_{I}(0)=0\end{cases}
$$

Note that $S(\cdot), I(\cdot), \varphi_{I}(\cdot)$ depend on $S_{0}, I_{0}$ and $u(t)$; however to simplify notations this dependence will not be stated explicitly. The signification of the parameters is as follows:

(1) the parameter $\beta>0$ is the transmission rate of the disease and the parameter $\gamma>0$ is the recovery rate;

(2) $S(t) \geq 0, I(t) \geq 0$ and $\int_{0}^{t} \gamma I(s) d s \geq 0$ are the proportions of individuals in the Susceptible, Infected and Recovered classes, respectively.

(3) $u:\left[0, \infty\left[\rightarrow\left[0, u_{\max }\right]\right.\right.$ is the vaccination rate; it is a measurable function; $u_{\max }<\infty$ is the maximal vaccination capacity. Therefore $\int_{0}^{t} u(s) d s$ is the proportion of people that vaccinated by the time $t$. Since $S(t)$ cannot be negative, this imposes some constraints on the set of admissible vaccination rates $u(t)$ (no more vaccination once $S(t)=0$ ). The admissible vaccinations rates (at the societal level) are:

$$
\mathcal{U}=\left\{u:\left[0, \infty\left[\rightarrow\left[0, u_{\max }\right] \mid u \text { measurable, } S(t) \geq 0 \forall t \geq 0\right\} .\right.\right.
$$


We also introduce the projection to $\mathcal{U}$. For any $u:\left[0, \infty\left[\rightarrow\left[0, u_{\max }\right]\right.\right.$ let $t_{u}$ be the largest value $t$ such that $u \cdot \mathbb{1}_{[0, t]}(\cdot) \in \mathcal{U}$. Then:

$$
\mathcal{R}(u):=u \cdot \mathbb{1}_{\left[0, t_{u}\right]}(\cdot)
$$

By convention for $t_{u}=\infty$ we take $\mathcal{R}(u):=u$ (in this case $u \in \mathcal{U}$ ).

(4) $\varphi_{I}(t)$ is the cumulative probability of infection in $[0, t]$ (in absence of vaccination). Its main use is to formulate the individual criterion for vaccination, see below equation (6).

We suppose that $\beta, \gamma, S_{0}>0$ and $I_{0}>0$ (with $S_{0}+I_{0} \leq 1$ ) are known and fixed from now on.

The first two equations of the system (1) represent the societal dynamics as illustrated in Figure 1; the individual dynamics is modeled by a continuous time Markov chain with individual jumping between the Susceptible, Infected, Recovered and Vaccinated classes (see Ref. 21 for the details of the Markov process).

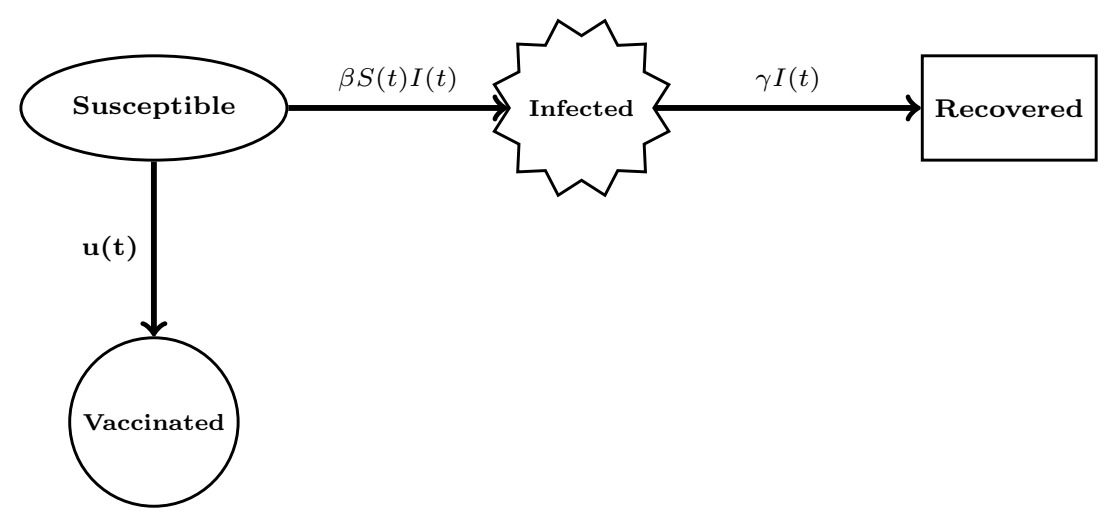

Fig. 1. Societal overall dynamics.

Moving from the Susceptible to the Vaccinated class depends only on the will of the individual. Moreover, his choice will not influence, on a global scale, the societal propagation dynamics. We suppose that each individual is aware of the propagation dynamics at the societal level (1). Therefore, the individual can choose between taking the risk of being vaccinated or taking the risk of contracting the disease. He makes a choice only according to his well-being. 
A coherence check of the model requires that the individual decisions sum up to give the overall vaccination dynamics (thus epidemic evolution). A natural question is whether such an equilibrium exists. We give a positive answer in a particular case that has not yet been considered in the literature.

\section{Optimal individual strategies}

The individual strategy is described by $\varphi_{V}(t)$ which is the probability of the individual to vaccinate in $[0, t]$. By convention $1-\varphi_{V}(\infty)$ is the probability to never vaccinate. Thus $\varphi_{V}$ is a cumulative distribution function (CDF) on $[0, \infty[\cup\{\infty\}$.

The requirement $u(t) \leq u_{\max }$ imposes some constraints on the CDF $\varphi_{V}$, see Ref. 21 for a detailed discussion. Let

$$
Z_{S(\cdot)}=\min _{\tau \geq 0}\{\tau \mid S(\tau)=0\}
$$

By convention if $S(\cdot)$ is always strictly positive we set $Z_{S(\cdot)}=\infty$. Two situations arise:

- On $\left[0, Z_{S(\cdot)}[\right.$ we can work, by density, with functions satisfying:

$$
\varphi_{V}^{\prime}(t)=\left(1-\varphi_{V}(t)\right) \lambda(t), \varphi_{V}(0)=0
$$

where $\lambda(\cdot)$ is a positive measurable function. The individual can choose his instantaneous vaccination rate $\lambda(\cdot)$; the bound $u_{\max }$ on the overall societal vaccination rate $u(t)$ induces a bound on $\lambda(t)$ of the form $\lambda(t) \leq$ $\frac{u_{\max }}{S(t)}$.

- On the other side, $\left.S(\cdot)\right|_{\left[Z_{S(\cdot)}, \infty[\right.} \equiv 0$ therefore on $\left[Z_{S(\cdot)}, \infty[\right.$ there are no susceptibles left to challenge the capacity of the vaccination centers and thus an additional individual arriving into such a population can vaccinate at any instant (s)he desires; consequently the function $\varphi_{V}$ is only required, as any CDF function, to be increasing, right-continuous and with $\varphi_{V}(\infty) \leq 1$.

In order to choose his best vaccination strategy the individual defines a cost, denoted $J_{\text {indi }}\left(\varphi_{V} ; u\right)$ :

$$
\begin{aligned}
& J_{\text {indi }}\left(\varphi_{V} ; u\right)=r_{I} \Phi_{I}(\infty)+\int_{0}^{\infty}\left[r_{I} "\left(\Phi(t)-\Phi_{I}(\infty)\right)\right. \\
& \left.+r_{V} e^{-\mathcal{D} t}\left(1-\varphi_{I}(t)\right)\right] d \varphi_{V}(t) .
\end{aligned}
$$


Here $\mathcal{D}>0$ is the discount factor, and $\Phi_{I}(t)$ is a function describing the risk of infection weighted according to the discount factor:

$$
\Phi_{I}(t)=\int_{0}^{t} e^{-\mathcal{D} s} \varphi_{I}^{\prime}(s) d s=\int_{0}^{t} e^{-\mathcal{D} s}\left(1-\varphi_{I}(s)\right) \beta I(s) d s, \Phi_{I}(0)=0 .
$$

The parameter $r_{V}$ is the cost of the vaccination (measured in money or as medical side-effects) and $r_{I}$ the cost of being infected.

Set:

$$
g(t)=r_{I} "\left(\Phi(t)-\Phi_{I}(\infty)\right)+r_{V} e^{-\mathcal{D} t}\left(1-\varphi_{I}(t)\right) .
$$

In particular $g(t) \in\left[-r_{I}, r_{I}+r_{V}\right], \forall t \geq 0$.

Thus, the individual wants to minimize $J_{i n d i}\left(\varphi_{V} ; u\right)=r_{I} \Phi_{I}(\infty)+$ $\int_{0}^{\infty} g(t) d \varphi_{V}(t)$, which, after elimination of the term $r_{I} \Phi_{I}(\infty)$ (independent of $\varphi_{V}$ ), amounts to looking for a minimum of

$$
\int_{0}^{\infty} g(t) d \varphi_{V}(t)=\int_{0}^{Z_{S(\cdot)}} g(t)\left(1-\varphi_{V}(t)\right) \lambda(t) d t+\int_{Z_{S(\cdot)}}^{\infty} g(t) d \varphi_{V}(t) .
$$

As in Ref. 21 the minimum of the second quantity is readily seen to be $-\left(1-\varphi_{V}\left(Z_{S(\cdot)}^{-}\right)\right) \cdot\left(\inf _{t \geq Z_{S(\cdot)}} g(t)\right)_{-} \quad$ (for any real number $x$, we denote $\left.x_{-}=\max \{-x, 0\}\right)$.

\subsection{The HJB equation associated to the individual optimal solutions}

Lemma 3.1. Let $u \in \mathcal{U}$. Then

(1) $I(t)$ vanishes to 0 at $\infty$ uniformly with respect to $u \in \mathcal{U}$ (but depending on $\left.\beta, \gamma, S_{0}, I_{0}\right)$;

(2) there exists $T_{\max }>0$ depending only on $\beta, \gamma, S_{0}, I_{0}$ such that the function $g(t)$ is positive on $\left[T_{\max }, \infty\left[\right.\right.$. In particular the optimal $d \varphi_{V}$ is null on $\left[T_{\max }, \infty[\right.$.

Proof. Let us compute the derivative of $g(t)$ :

$$
\begin{aligned}
g^{\prime}(t) & =r_{I} e^{-\mathcal{D} t}\left(1-\varphi_{I}(t)\right) \beta I(t)-\mathcal{D} r_{V} e^{-\mathcal{D} t}\left(1-\varphi_{I}(t)\right) \\
& -r_{V} e^{-\mathcal{D} t}\left(1-\varphi_{I}(t)\right) \beta I(t) \\
& =e^{-\mathcal{D} t}\left(1-\varphi_{I}(t)\right)\left[\left(r_{I}-r_{V}\right) \beta I(t)-r_{V} \mathcal{D}\right] .
\end{aligned}
$$

The second equation in (1) shows that $I(t)$ can be either decreasing (if $S_{0} \leq \gamma / \beta$ ) or increasing while $S(t) \geq \gamma / \beta$ and then decreasing. In 
particular $I(t)$ can be larger than $\frac{r_{V} \mathcal{D}}{\left(r_{I}-r_{V}\right) \beta}$ at most on some bounded interval (depending on $S_{0}, I_{0}, u$ ). Thus in general $g(t)$ has the form in Figures 2 and 3: decreasing, then increasing then decreasing again. Moreover $\lim _{t \rightarrow \infty} g(t)=0$.

The optimal $d \varphi_{V}$ is null on any interval $[T, \infty[$ such that $g(t)$ is decreasing (thus positive) on the whole interval. Therefore it is enough to find $T_{\max }$ such that $I(t) \leq \frac{r_{V} \mathcal{D}}{\left(r_{I}-r_{V}\right) \beta}$ for $t \geq T_{\max }$. To this end we will prove that $I(t)$ vanishes at infinity uniformly with respect to $u$ (but depending on $S_{0}, I_{0}, \gamma$ and $\beta$ ).

Let $C \leq I_{0}$ and $T_{C}=\frac{\ln \left(\frac{I_{0}}{C}\right)+\frac{S_{0}}{C}}{\gamma}>0$, chosen such that $I_{0} e^{-\gamma t+\frac{S_{0}}{C}} \leq$ $C, \forall t \geq T_{C}$. Suppose, by contradiction, that there exists $t^{*} \geq T_{C}$ with $I\left(t^{*}\right)>C$ (note that $t^{*}$ may depend on $u$ ). Thus, as $I$ is continuous and $\lim _{t \rightarrow \infty} I(t)=0$, we find $t^{* *} \geq t^{*} \geq T_{C}$ such that $I\left(t^{* *}\right)=C$. But, since $C \leq I_{0}$, we also have that $I\left(t^{* *}\right)=C \leq I_{0}$ implies $I(t) \geq C, \forall t \leq t^{* *}$ and thus $I(t) \geq C, \forall t \leq T_{C}$.

On the other side, $S^{\prime}(t) \leq-\beta S(t) I(t)$ thus $S(t) \leq S_{0} e^{-\beta t C}$, $\forall t \leq T_{C}$. Moreover $I^{\prime}(t)=(\beta S(t) I(t)-\gamma I(t))$ implies : $I(t) \leq$ $I_{0} e^{-\gamma t+S_{0} \beta \int_{0}^{t} e^{-\beta C \tau} d \tau}<I_{0} e^{-\gamma t+\frac{S_{0}}{C}} \leq C$, which is absurd.

Thus for any $C$ small enough $\left(C \leq I_{0}\right)$, there exists $T_{C}>0$ independent of $u$ such that $I(t) \leq C, \forall t \geq T_{C}$ which means that $I$ vanishes at infinity independently of $u$. This gives the first point of the result.

The second point follows from the behavior of the function $g(t)$ and the definition of the individual cost.

Remark 3.1. The point 1 of Lemma 3.1 can also be proved in the following way: for any $\varepsilon<I_{0}$ let $t_{\varepsilon}$ be the unique point such that $I\left(t_{\varepsilon}\right)=\varepsilon$ (unique because $I(t)$ can only decrease once it is below $\left.I_{0}\right)$. Then for any $t \leq t_{\varepsilon}$ we have $I(t) \geq I\left(t_{\varepsilon}\right)=\varepsilon$. But since

$$
1 \geq \int_{0}^{\infty} \gamma I(t) d t \geq \int_{0}^{t_{\varepsilon}} \gamma I(t) d t \geq \gamma I\left(t_{\varepsilon}\right) t_{\varepsilon},
$$

one obtains $t_{\varepsilon} \leq \frac{1}{\gamma \varepsilon}$ thus any $t \geq \frac{1}{\gamma \varepsilon}$ has $I(t) \leq I\left(t_{\varepsilon}\right)=\varepsilon$.

Note that on $\left[Z_{S(\cdot)}, \infty[\right.$ the variable $I(t)$ can only decrease. Thus, considering the arguments of Lemma 3.1 the function $g(t)$ can be increasing and then decreasing or only decreasing. In both situations, the minimum value, if it is negative, is attained at point $Z_{S(\cdot)}$ (otherwise it is null). Thus:

$$
-\left(\inf _{t \geq Z_{S(\cdot)}} g(t)\right)_{-}=-\left(g\left(Z_{S(\cdot)}\right)\right)_{-} .
$$


The individual minimization problem can therefore be rephrased as minimizing

$$
\int_{0}^{\infty} g(t) d \varphi_{V}(t)=\int_{0}^{Z_{S(\cdot)}} g(t)\left(1-\varphi_{V}(t)\right) \lambda(t) d t-\left(1-\varphi_{V}\left(Z_{S(\cdot)}^{-}\right) \cdot\left(g\left(Z_{S(\cdot)}\right)\right)_{-} .\right.
$$

For any $t \geq 0$ we introduce the notation

$V(t):=\inf \left\{\int_{t}^{\infty} g(\tau) d \varphi_{V}(\tau) \mid \varphi_{V}\right.$ increasing, right continuous with left limits, $\varphi_{V}(t)=0, \varphi_{V}(\infty) \leq 1, \varphi_{V}^{\prime}(\tau)=\left(1-\varphi_{V}(\tau)\right) \lambda(\tau), \forall \tau \in\left[t, Z_{S(\cdot)}[\right.$ with $\left.0 \leq \lambda(\tau) \leq u_{\max } / S(\tau)\right\}$.

Previous arguments show that for $t \in\left[0, Z_{S(\cdot)}[\right.$

$$
\begin{aligned}
& V(t):=\inf \left\{\int_{t}^{Z_{S(\cdot)}} g(\tau)\left(1-\varphi_{V}(\tau)\right) \lambda(\tau) d \tau-\left(1-\varphi_{V}\left(Z_{S(\cdot)}^{-}\right)\right) \cdot\left(g\left(Z_{S(\cdot)}\right)\right)_{-} \mid\right. \\
& \varphi_{V}(t)=0, \varphi_{V}(\infty) \leq 1, \varphi_{V}^{\prime}(\tau)=\left(1-\varphi_{V}(\tau)\right) \lambda(\tau), \forall \tau \in\left[t, Z_{S(\cdot)}[\text { with }\right. \\
& \left.0 \leq \lambda(\tau) \leq u_{\max } / S(\tau)\right\} .
\end{aligned}
$$

Standard manipulations allow to see that $V$ is continuous on $\left[0, Z_{S(\cdot)}[\cup] Z_{S(\cdot), \infty[}\right.$,

Moreover the limit $\lim _{t} Z_{S(\cdot)} V(t)$ equals $-\left(g\left(Z_{S(\cdot)}\right)\right)_{-}$and can be attained with a $\varphi_{V}$ that is continuous. To see this, consider the relevant case $Z_{S(\cdot)}<\infty$ and treat first the situation when $g\left(Z_{S(\cdot)}\right) \geq 0$. Then in the limit $t \nearrow Z_{S(\cdot)}$ the best possible value for $V(t)$ is null, attained with $\varphi_{V}=0$. When $g\left(Z_{S(\cdot)}\right)<0$, since $g(\cdot)$ is continuous and the total mass of $\varphi_{V}$ cannot be larger than one, in the limit $t \nearrow Z_{S(\cdot)}$ the best possible value for $V(t)$ is $g\left(Z_{S(\cdot)}\right)$, attained for the law obtained setting $\lambda(\tau)=u_{\max } / S(\tau)$. To see that in this case the mass is one it is enough to prove that $\varphi_{V}\left(Z_{S(\cdot)}^{-}\right)=1$, or equivalently $\int_{t}^{Z_{S(\cdot)}} u_{\max } / S(\tau) d \tau=\infty$; the equation of $S^{\prime}(t)$ integrated between $Z_{S(\cdot)}-\epsilon$ and $Z_{S(\cdot)}$ gives the upper bound $S\left(Z_{S(\cdot)}-\epsilon\right) \leq \epsilon u_{\max }$ and the conclusion follows.

Thus setting $V\left(Z_{S(\cdot)}\right)=-\left(g\left(Z_{S(\cdot)}\right)\right)_{-}$we obtain that $V(\cdot)$ is continuous on $\left[0, Z_{S(\cdot)}\right]$ and is a viscosity solution of the following Hamilton Jacobi Bellman equation (see Ref. 25):

$$
\begin{aligned}
& -\left[V^{\prime}(t)+\min _{\lambda(t) \in\left[0, u_{\max } / S(t)\right]}(g(t)-V(t)) \lambda(t)\right]=0, t<Z_{S(\cdot)} \\
& V\left(Z_{S(\cdot)}\right)=-\left(g\left(Z_{S(\cdot)}\right)\right)_{-}
\end{aligned}
$$


On the other hand, for $t \geq Z_{S(\cdot)}: V(t)=-(g(t))_{-}$and also $V(t)=0$ for any $t \geq T_{\max }$ which means that on $\left[Z_{S(\cdot)}, T_{\max }\right.$ [ (if it is non-void) the value function $V$ is solution of the HJB equation

$$
\begin{aligned}
& -\min \left\{V^{\prime}(t), g(t)-V(t)\right\}=0, Z_{S(\cdot)} \leq t<T_{\max } \\
& V\left(T_{\max }\right)=0 .
\end{aligned}
$$

Combining equations(16)-(17) and (18)-(19) we obtain that $V$ is the continuous viscosity solution of:

$\forall t \leq T_{\max }:-\min \left\{V^{\prime}(t), V^{\prime}(t) S(t)+u_{\max }(g(t)-V(t))\right\}=0, V\left(T_{\max }\right)=0$.

Note that, for any given $g(\cdot), S(\cdot)$, the problem (20) can be written

$$
-F_{g(\cdot), S(\cdot)}\left(t, V, V^{\prime}\right)=0, V\left(T_{\max }\right)=0 .
$$

where $F_{g(\cdot), S(\cdot)}:\left[0, T_{\max }\right] \times \mathbb{R} \times \mathbb{R} \rightarrow \mathbb{R}$ is defined as

$$
F_{g(\cdot), S(\cdot)}(t, v, p)=\min \left\{p, p S(t)+u_{\max }(g(t)-v)\right\} .
$$

In particular $F_{g(\cdot), S(\cdot)}$ is continuous.

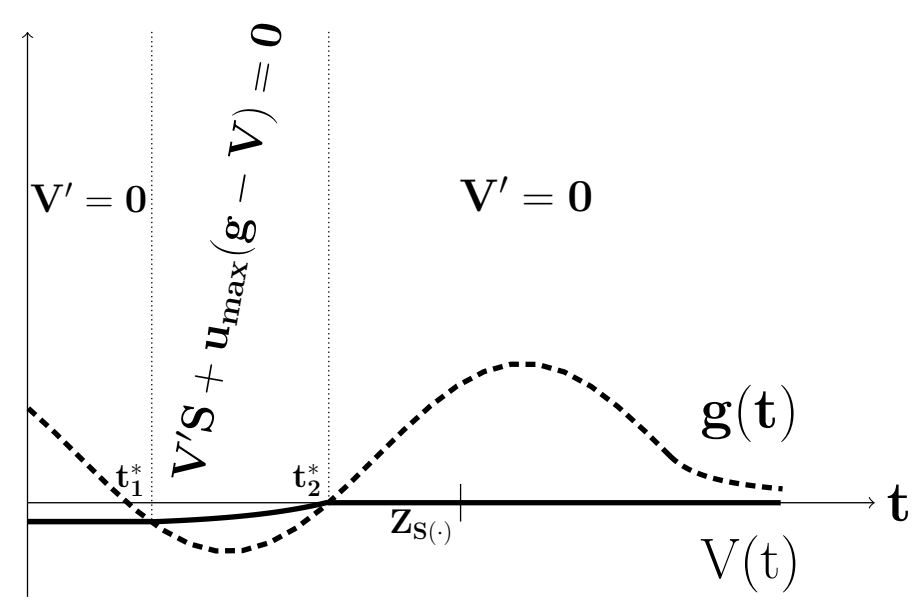

Fig. 2. Representation of the functions $g(t)$ (dashed line) and $V(t)$ (solid line) for the situation $g(t) \geq 0$ for all $t \geq Z_{S(\cdot)}$. In this case the equation of $V$ can either be $V^{\prime}=0$ or $V^{\prime} S+u_{\max }(g-V)=0$. 


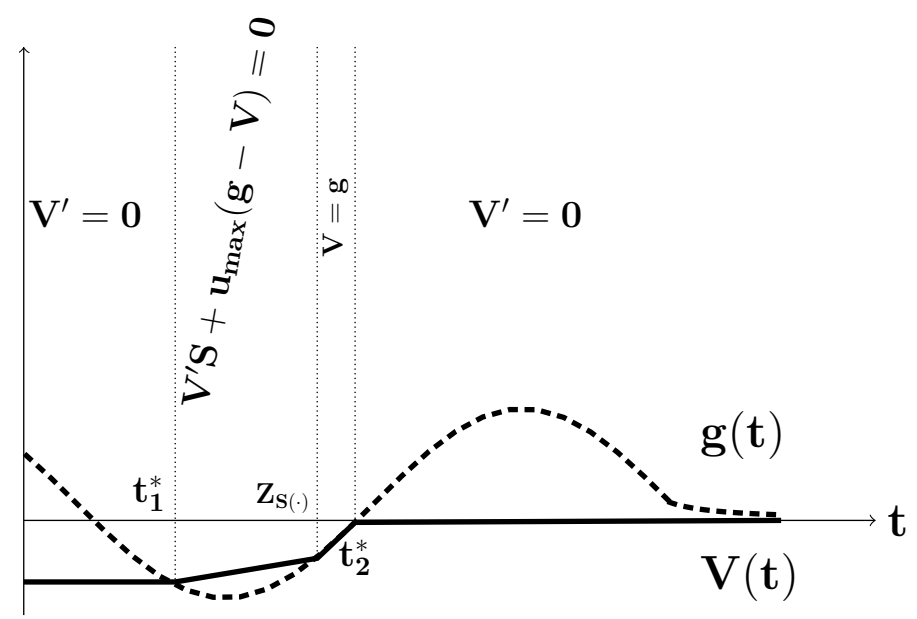

Fig. 3. Representation of the functions $g(t)$ (dashed line) and $V(t)$ (solid line) for the situation $g\left(Z_{S(\cdot)}\right)<0$. With respect to the Figure 2 a new region appears, for $t \in\left[Z_{S(\cdot)}, t_{2}^{*}\right]$ where $V=g$.

\subsection{Structure of individual optimal solutions}

The considerations of the previous section allow to prove:

Lemma 3.2. Let $u$ be an arbitrary control in $\mathcal{U}$ and denote by $S(\cdot)$ and $g(\cdot)$ the corresponding solutions of (1). Then the value function $V(\cdot)$ defined in (14) is the unique solution of the HJB equation (20). Moreover $V(0)$ is attained by at least one optimal strategy $\varphi_{V}$ having the following form:

(1) If $\inf _{t \geq 0} g(t)=0$ then $V(0)=0$ and $\varphi_{V}=0$;

(2) If $\inf _{t \geq 0} g(t)<0$, let $t_{u} \leq T_{\max }$ be such that $g\left(t_{u}\right)=\inf _{t \geq 0} g(t)$. If $t_{u} \geq Z_{S(\cdot)}$ then $d \varphi_{V}=\delta_{t_{u}}$ and $V(0)=g\left(t_{u}\right)$;

(3) If $t_{u}<Z_{S(\cdot)}$ there exist $t_{u}^{1}$, $t_{u}^{2}$ with $0 \leq t_{u}^{1} \leq t_{u}<t_{u}^{2} \leq \min \left\{Z_{S(\cdot)}, T_{\max }\right\}$ such that:

$$
\left\{\begin{array}{l}
d \varphi_{V}(t)=\left(1-\varphi_{V}\right) \frac{u_{\max }}{S(t)} d t, t \in\left[t_{u}^{1}, t_{u}^{2}[\right. \\
d \varphi_{V}(t)=0, t \notin\left[t_{u}^{1}, t_{u}^{2}[.\right.
\end{array}\right.
$$

In this case $\inf _{t \geq 0} g(t)<V(0)<0$.

Proof. The considerations in Section 3.1 ensure that the value function is solution of (20). To prove the uniqueness, we apply the theorem 2.4 page 26 from Ref. 26 (see also chapter II. 3 from Ref. 27 and the equation 
2.20 section II.2 from Ref. 28). To this end consider $W(t)=e^{t} V(t)$, thus $V^{\prime}(t)=e^{-t}\left(W^{\prime}(t)-W(t)\right)$. We replace $V(t)$ and $V^{\prime}(t)$ in $(20)$ and obtain that $W(t)$ is solution of the following problem:

$$
\begin{aligned}
& \forall t \leq T_{\max }: \\
& -\min \left\{W^{\prime}(t)-W(t),\left(W^{\prime}(t)-W(t)\right) S(t)+u_{\max }\left(e^{t} g(t)-W(t)\right)\right\}=0, \\
& W\left(T_{\max }\right)=0 .
\end{aligned}
$$

The uniqueness of the solution of (24) is obtained under assumptions of strict monotonicity of $F_{W}(t, v, p)=-\min \left\{p-v,(p-v) S(t)+u_{\max }\left(e^{t} g(t)-\right.\right.$ $v)\}$ with respect to its second argument. In practice one has to prove

$$
\begin{array}{r}
\forall t \geq 0, \forall p \in \mathbb{R}, \forall v_{1} \geq v_{2}: F_{W}\left(t, v_{1}, p\right)-F_{W}\left(t, v_{2}, p\right) \geq C_{m}\left(v_{1}-v_{2}\right), \\
\text { with } C_{m}>0 .
\end{array}
$$

In our case, $F_{W}$ satisfies $(25)$ with $C_{m}=\min \left(1, u_{\max }\right)>0$.

To prove the second part of the result, we propose a solution candidate $\mathcal{V}$. First of all, $\mathcal{V}(t):=-(g(t))_{-}$on $\left[\min \left\{Z_{S(\cdot)}, T_{\max }\right\}, \infty[\right.$. In this case $d \varphi_{V}$ is either null or a Dirac mass (depending on whether $g$ is positive or negative).

For $t<\min \left\{Z_{S(\cdot)}, T_{\max }\right\}$ two alternatives appear:

- When $Z_{S(\cdot)}<\infty$ and $g\left(Z_{S(\cdot)}\right)<0$ then $S(t) \mathcal{V}^{\prime}(t)+u_{\max }(g(t)-\mathcal{V}(t))=0$ on some interval $\left[t_{1}^{*}, Z_{S(\cdot)}\right.$ [ with $t_{1}^{*}$ the first (solving backwards from $\left.Z_{S(\cdot)}\right)$ such that $\mathcal{V}\left(t_{1}^{*}\right)=g\left(t_{1}^{*}\right)$. In this case $d \varphi_{V}=\left(1-\varphi_{V}\right) u_{\max } / S(t)$ on some interval and null elsewhere. On $\left[0, t_{1}^{*}\right]$, the function $\mathcal{V}(t)$ is constant.

- For other situations, denote

$$
t_{2}^{*}=\inf \{t \geq 0 \mid g(\tau) \geq 0, \forall \tau \geq t\} .
$$

Then $S(t) \mathcal{V}^{\prime}(t)+u_{\max }(g(t)-\mathcal{V}(t))=0$ on some interval $\left[t_{1}^{*}, t_{2}^{*}\left[\right.\right.$ with $t_{1}^{*}$ the first (solving backwards from $t_{2}^{*}$ ) such that $\mathcal{V}\left(t_{1}^{*}\right)=g\left(t_{1}^{*}\right)$. In this case $d \varphi_{V}=\left(1-\varphi_{V}\right) u_{\max } / S(t)$ on some interval and null elsewhere. On $\left[0, t_{1}^{*}\right]$, the function $\mathcal{V}(t)$ is constant.

Elementary computations allow to prove that $\mathcal{V}$ is a solution of (20), therefore the unique solution, therefore $\mathcal{V}=V$. In particular, this implies the alternatives 1-3. See Figures 2 and 3 for an illustration.

\subsection{Equilibrium}

An equilibrium between the individual vaccination and the societal vaccination satisfies $u(t)=S(t) \lambda(t)$ (see Ref. 21). The Lemma 3.2 suggests 
that an equilibrium is necessarily of the form $u(t)=u_{\max } \mathbb{1}_{\left[T_{1}^{*}, T_{2}^{*}\right]}(t)$ with $T_{1}^{*}, T_{2}^{*} \leq T_{\max }$.

To prove the existence of an equilibrium we introduce $K=\left\{\left(t_{1}, t_{2}\right) \in\right.$ $\left.\left[0, T_{\text {max }}\right]^{2} \mid t_{1} \leq t_{2}\right\}$ and the function $\mathcal{F}: K \rightarrow 2^{K}\left(2^{K}\right.$ are the sub-ensembles of $K)$ defined as follows. For any $x=\left(T_{1}, T_{2}\right) \in K$ consider $V^{x}$ the solution of the HJB equation (20) corresponding to $u^{x}(t)=\mathcal{R}\left(u_{\max } \mathbb{1}_{\left[T_{1}, T_{2}\right]}(\cdot)\right)$. If, with the notations of the Lemma 3.2 , the alternative 3 arises then set $\mathcal{F}(x)=\left\{\left(t_{u^{x}}^{1}, t_{u^{x}}^{2}\right)\right\}$; otherwise set $\mathcal{F}(x)=\left\{(t, t) \mid 0 \leq t \leq T_{\text {max }}\right\}$.

Theorem 3.1. An equilibrium strategy exists. It is of the form $u^{e}=$ $u_{\max } \mathbb{1}_{\left[T_{1}^{e}, T_{2}^{e}\right]}(\cdot),\left(T_{1}^{e}, T_{2}^{e}\right) \in K$.

Proof. An equilibrium corresponds to a fixed point of $\mathcal{F}$. Moreover the definition (3) and equation (11) ensure that any point $x^{*}$ with $x^{*} \in \mathcal{F}\left(x^{*}\right)$ corresponds necessarily to an admissible control (see also the arguments that allow to prove the continuity of $V$ defined in equation (15)). We use the Kakutani fixed-point theorem and will check its assumptions:

- for all $x \in K, \mathcal{F}(x)$ is a non-empty convex subset of $K$;

- the graph of the set-valued function $\mathcal{F}$ is closed in $K \times K$.

The first property follows from the definition of $\mathcal{F}$. For the second consider two sequences $\left(x_{n}=\left(T_{1}^{n}, T_{2}^{n}\right)\right)_{n \geq 0},\left(y_{n}\right)_{n \geq 0} \subset K$ such that $y_{n} \in$ $\mathcal{F}\left(x_{n}\right), x_{n} \rightarrow x=\left(T_{1}, T_{2}\right), y_{n} \rightarrow y$. We have to prove that $y \in \mathcal{F}(x)$. Denote $u^{x}=u_{\max } \mathbb{1}_{\left[T_{1}, T_{2}\right]}(\cdot), u^{x_{n}}=u_{\max } \mathbb{1}_{\left[T_{1}^{n}, T_{2}^{n}\right]}(\cdot)$.

We use the following stability properties from Ref. 25 page 176 : let $\left(V_{n}\right)_{n \geq 1}$ satisfy (in the viscosity sense):

$$
-F_{n}\left(t, V_{n}(t), V_{n}^{\prime}(t)\right)=0, t \leq T_{\max }, V_{n}\left(T_{\max }\right)=0 .
$$

Suppose that the sequence $\left(F_{n}\right)_{n \geq 1}$ converges uniformly on compact sets to some $F$ and the sequence $\left(V_{n}\right)_{n \geq 1}$ converges to $V$ in $C\left(\left[0, T_{\max }\right]\right)$. Then $V$ is a viscosity solution of

$$
-F\left(t, V(t), V^{\prime}(t)\right)=0, t \leq T_{\max }, V\left(T_{\max }\right)=0 .
$$

Here we take, $F=F_{g(\cdot), S(\cdot)}, S, g, I$ correspond to the control $\mathcal{R}\left(u^{x}\right)$; $F_{n}=F_{g_{n}(\cdot), S_{n}(\cdot)}, S_{n}, g_{n}, I_{n}$ correspond to the control $\mathcal{R}\left(u^{x_{n}}\right)$.

We use the a priori estimate (16) of the theorem 2.1 from Ref. 29 which asserts that, denoting $U(t)=\int_{0}^{t} \mathcal{R}\left(u^{x}\right)(\tau) d \tau, U_{n}(t)=\int_{0}^{t} \mathcal{R}\left(u^{x_{n}}\right)(\tau) d \tau$, there exists a constant $C$ such that:

$\left|S(t)-S_{n}(t)\right|+\int_{0}^{t}\left|S(\tau)-S_{n}(\tau)\right| d \tau \leq C\left[\left|U(t)-U_{n}(t)\right|+\int_{0}^{t}\left|U(\tau)-U_{n}(\tau)\right| d \tau\right]$, 
for any $t \in\left[0, T_{\max }\right]$. The same works for $I(\cdot)$. But the convergence of $T_{1}^{n}$ to $T_{1}$ and $T_{2}^{n}$ to $T_{2}$ implies the uniform convergence of $U_{n}$ to $U$ and thus uniform convergence of $S_{n}$ to $S, I_{n}$ to $I$; hence $g_{n}$ converges to $g$ uniformly. This is enough to ensure the uniform convergence of $F_{n}$ to $F$. By Lemma 3.2 to any solution of equations (28) and (27) we can associate an element of $K$; using the compactness of $K$, we obtain the conclusion.

\section{References}

1. R. Zeckhauser and D. Shepard, Where now for saving lives?, Law and Contemporary Problems 40, 5 (1976).

2. S. Anand and K. Hanson, Disability-adjusted life years: a critical review, Journal of Health Economics 16, 685 (1997).

3. F. Sassi, Calculating QALYs, comparing QALY and DALY calculations, Health Policy and Planning 21, 402 (2006).

4. H. W. Hethcote and P. Waltman, Optimal vaccination schedules in a deterministic epidemic model, Mathematical Biosciences 18, 365 (December 1973).

5. A. Abakuks, Optimal immunisation policies for epidemics, Advances in Appl. Probability 6, 494 (1974).

6. R. Morton and K. H. Wickwire, On the optimal control of a deterministic epidemic, Advances in Appl. Probability 6, 622 (1974).

7. S. P. Sethi and P. W. Staats, Optimal control of some simple deterministic epidemic models, J. Oper. Res. Soc. 29, 129 (1978).

8. O. Diekmann and J. Heesterbeek, Mathematical epidemiology of infectious diseases. Model building, analysis and interpretation. (Wiley Series in Mathematical and Computational Biology. Chichester: Wiley., 1999).

9. L. Laguzet and G. Turinici, Global optimal vaccination in the SIR model: Properties of the value function and application to costeffectiveness analysis, Mathematical Biosciences 263, 180 (2015).

10. C. T. Bauch, A. P. Galvani and D. J. D. Earn, Group interest versus self-interest in smallpox vaccination policy, Proceedings of the National Academy of Sciences 100, 10564 (2003).

11. P. J. Francis, Optimal tax/subsidy combinations for the flu season, Journal of Economic Dynamics and Control 28, 2037 (2004).

12. B. Buonomo, A. dOnofrio and D. Lacitignola, Global stability of an $\{$ SIR $\}$ epidemic model with information dependent vaccination, Mathematical Biosciences 216, 9 (2008). 
13. A. dOnofrio, P. Manfredi and E. Salinelli, Vaccinating behaviour, information, and the dynamics of SIR vaccine preventable diseases, Theoretical Population Biology 71, 301 (2007).

14. A. P. Galvani, T. C. Reluga and G. B. Chapman, Long-standing influenza vaccination policy is in accord with individual self-interest but not with the utilitarian optimum, Proceedings of the National Academy of Sciences 104, 5692 (2007).

15. T. C. Reluga, C. T. Bauch and A. P. Galvani, Evolving public perceptions and stability in vaccine uptake, Math. Biosci. 204, 185 (2006).

16. M.-G. Cojocaru, Dynamic equilibria of group vaccination strategies in a heterogeneous population, Journal of Global Optimization 40, 51 (2008).

17. F. H. Chen, Modeling the effect of information quality on risk behavior change and the transmission of infectious diseases, Mathematical Biosciences 217, 125 (2009).

18. P. E. M. Fine and J. A. Clarkson, Individual versus public priorities in the determination of optimal vaccination policies, American Journal of Epidemiology 124, 1012 (1986).

19. D. L. Brito, E. Sheshinski and M. D. Intriligator, Externalities and compulsary vaccinations, Journal of Public Economics 45, 69 (1991).

20. S. Bhattacharyya and C. T. Bauch, Wait and see vaccinating behaviour during a pandemic: A game theoretic analysis, Vaccine 29, 5519 (2011).

21. L. Laguzet and G. Turinici, Individual vaccination as Nash equilibrium in a SIR model with application to the 2009-10 Influenza A(H1N1) epidemic in France, Bulletin of Mathematical Biology in press, p. 30pp (2015), doi:10.1007/s11538-015-0111-7.

22. D. A. Gomes, J. Mohr and R. R. Souza, Continuous time finite state mean field games, Applied Mathematics \& Optimization 68, 99 (2013).

23. O. Guant, Existence and uniqueness result for mean field games with congestion effect on graphs, Applied Mathematics $\&$ Optimization 72, 291 (2015).

24. R. M. Anderson and R. M. May, Infectious Diseases of Humans Dynamics and Control (Oxford University Press, 1992).

25. A. Bressan and B. Piccoli, Introduction to the mathematical theory of control, AIMS Series on Applied Mathematics, Vol. 2 (American Institute of Mathematical Sciences (AIMS), Springfield, MO, 2007).

26. G. Barles, Solutions de viscosité des équations de Hamilton-Jacobi, Mathématiques \& Applications (Berlin) [Mathematics \& Applications], Vol. 17 (Springer-Verlag, Paris, 1994). 
27. M. Bardi and I. Capuzzo-Dolcetta, Optimal control and viscosity solutions of Hamilton-Jacobi-Bellman equations Systems\&Control:Foundations \& Applications, Systems \& Control: Foundations \& Applications "(Birkhäuser Boston Inc., Boston, MA, 1997), With appendices by Maurizio Falcone and Pierpaolo Soravia.

28. M. G. Crandall and P.-L. Lions, Viscosity solutions of Hamilton-Jacobi equations, Trans. Amer. Math. Soc. 277, 1 (1983).

29. A. Bressan, JR. and F. Rampazzo, Impulsive control systems with commutative vector fields., J. Optim. Theory Appl. 71, 67 (1991). 